\title{
XIII.
}

Aus der königlichen chirurgischen Universitätsklinik zu Bonn. (Direktor Prof. Dr. Bi er.)

\section{Beitrag zur Kenntnis der Osteomalacia chronica deformans hypertrophica. (Paget.) \\ Von}

Dr. Viktor Schmieden, Assistent der Klinik, Privatdozent für Chirurgie.

(Mit 4 Abbildungen im Text und Tafel III.)

Die folgenden Zeilen sollen zu der im Titel genannten, sehr seltenen Krankbeitsform einen Beitrag liefern, der besonders dadurch von Wert sein dürfte, weil es möglich war, pathologisch-histologische Untersuchungen der Erkrankung beizubringen, und weil ferner der Bau des Knochens einen Rückschlub auf pathologische Knochenwachstumsvorgänge im allgemeinen gestattete. Paget hat als erster im Jahre 1876 auf Grund einiger Fälle die vorliegende Krankheit als eine Erkrankung sui generis erkannt und damit die Reihe von Publikationen eröffnet, die sich an seine Ausführungen anschlob. Bei der Seltenheit des Leidens dürfte auf den ersten Blick ein Befund überraschen, wie er in Fig. 1 und 2 photographisch wiedergegeben ist; eine auf Lues in keiner Weise verdächtige 58jährige Frau erkrankte spontan an einem sich langsam entwickelnden Riesenwuchs eines einzelnen Knochens und zwar des rechten Schienbeins. Der erste Anfang des Leidens lag 3 Jahre zurück, eine Ursache war unbekannt. Langsam zunehmende Beschwerden, besonders heftige rheumatoide Schmerzen, erschwerten das Gehen dauernd mehr und mehr, und führten sie endlich ins Krankenhaus; das Kniegelenk wurde immer steifer. Schon die flüchtige Untersuchung zeigt, daß nur die Tibia krank ist; sie ist in allen Dimensionen stark vergrößert, dabei nach vorn und innen bogenförmig verkrümmt, so daß bei aufrechter Stellung, wie Fig. 1 zeigt, bei nebeneinander stehenden 
Füßen die Kniee weit auseinanderstehen. Die Epiphysen, besonders die obere, mißgestaltet und verdickt, paßt nur noch schlecht in die Gelenkverbindung mit dem Femur, und ruft daher heftige Funktionsstörung im Kniegelenk hervor. Die Haut über dem kranken Unterschenkel ist inselförmig braun pigmentiert (vergl. Fig. 1), und dabei auffallend glatt und zart. Die übrigen Knochen

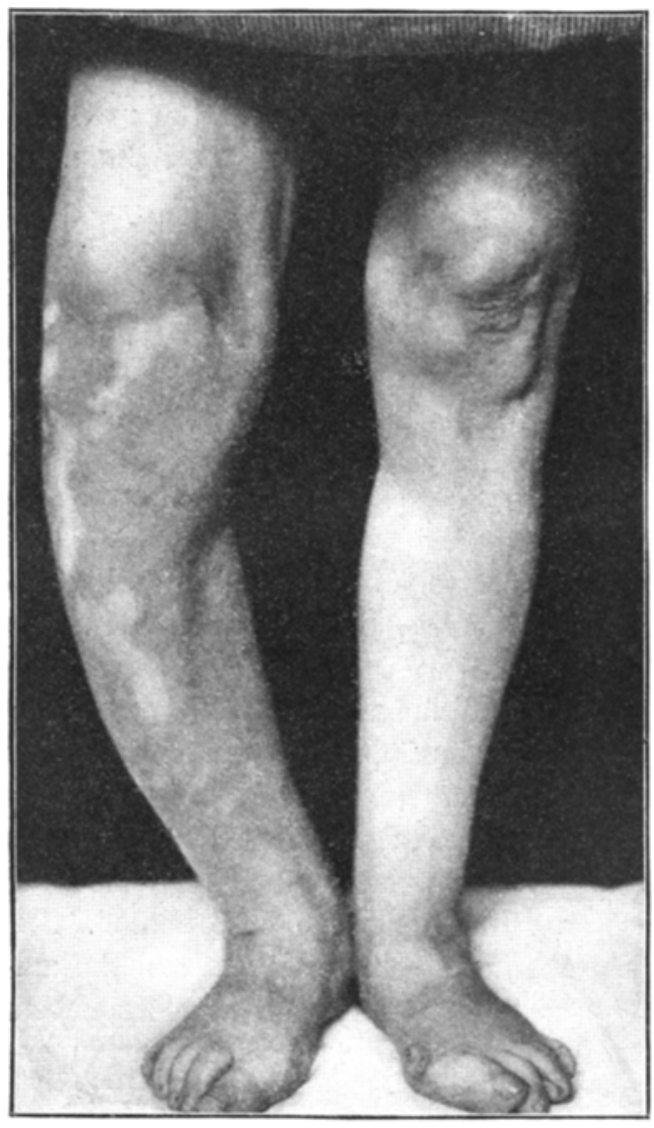

Fig. 1. des Körpers sind frei von nachweisbarer Er. krankung; insbesondere fehlt jede Veränderung an den angrenzenden Knochen, Fibula, Femur und Fußwurzelknochen.

Die aus der Untersuchung gewonnene Anschauung wurde durch das Röntgenbild bestätigt. An der abnormen Bildung ist lediglich die Tibia beteiligt; sie wölbt sich in der Seitenansicht bogenförmig über die normale Fibula, die als Sehne über den Bogen gespannt ist. Sofort aber fällt auf dem Radiogramm (Fig. 3) die eigenartige Architektur der Spongiosa auf.. Der ganze Innenraum der Tibia besteht aus spitzbogenförmig angeordneten Balken, an denen man auf den ersten Blick

erkennt, daß sie unter den abnormen Belastungsverhältnissen auf dem Wege funktioneller Anpassung entstanden sind. Diese Transformation der Spongiosa gehört zu dem Interessantesten, was der Fall bietet und wird uns bei der mikroskopischen Beschreibung noch zu beschäftigen haben.

Wie gesagt, hat Paget uns diese Krankheit zum ersten Male 
beschrieben und unser Fall entspricht bis aufs letzte seinem Symptomenkomplex. Nicht allein die Krümmungsrichtung des Schienbeins, die abgerundete Crista, die langsam entstehenden leftigen Schmerzen, die spezifische Veränderung der Haut stimmten ganz mit seinem ersten, grundlegenden Fall ïberein, sondern auch die Arthritis deformans der Nachbargelenke, die seit Paget mehr. fach konstatiert wurde, fehlt in unserem Falle nicht. Wenn also die Pag etsche Untersuchung die vorliegende Krankheit scharf von anderen, insbesondere von chronischen luetischen Schienbeinverkrümmungen abtrennt, so darf andererseits nicht vergessen werden, dali es sich hier um ein Krankheitsbild handelt, das uns im übrigen sehr wobl bekannt ist und das die mannigfachsten Ursachen haben kann, die sich aber alle von einem gemeinsamen Gesichtspunkt aus betrachten lassen. Wir wissen, dal chronische

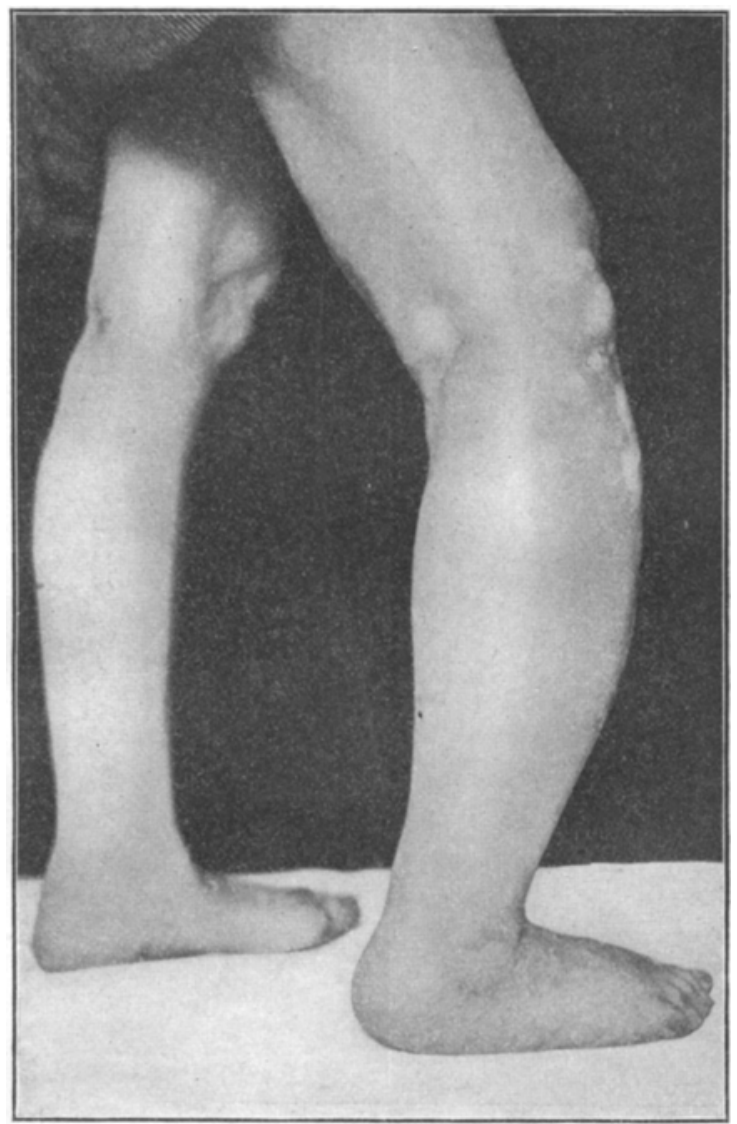

Fig. 2.

Entzündungsvorgänge ähnliche Veränderungen am Schienbein erzeugen können: chronische Osteomyelitis, Ulcera cruris, wenn sie auf den Knochen übergehen, unter Umständen auch Geschwülste oder abnorm heilende Frakturen, nicht zuletzt die chronische gummöse Ostitis; sie alle regen im Mark der Tibia ähnlichen Riesenwuchs gelegentlich an; endlich sind auch bei ehronischen Krankheitszuständen, insbesondere 
Reizungszuständen des Kniegelenks, Gelenkmäusen usw., ähnliche Knochenvergrößerungen konstatiert. Die miskroskopische Untersuchung zeigt in unserem Fall, dal auch hier nichts anderes ein chronisch entzündlicher Vorgang, der in ganz diffuser Weise den Schaft und die obere Epiphyse der Tibiatergriffen hat. Aus der Vorgeschichte der Kranken bedarf

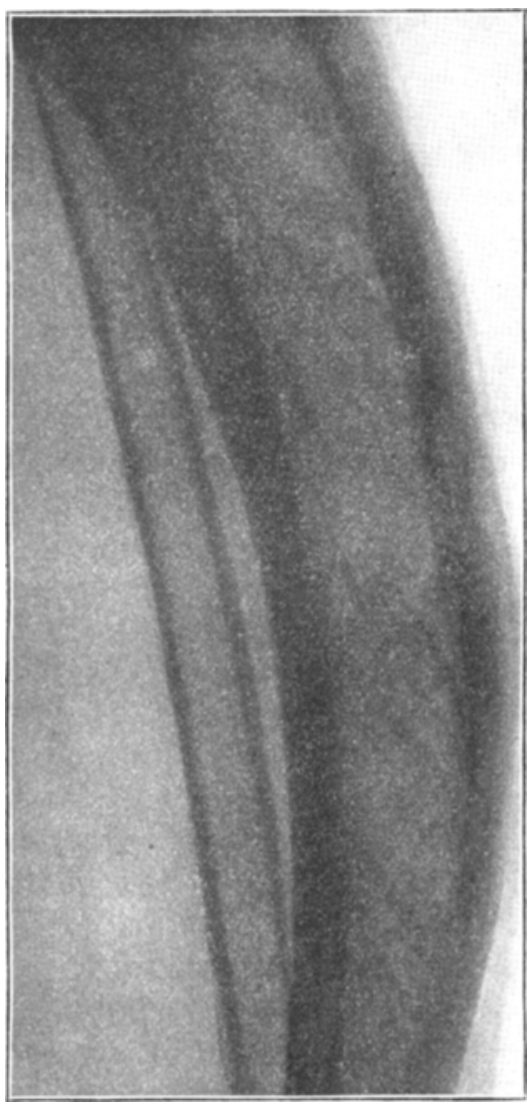

Fig. 3. nur der Umstand der Erwähnung, daß antiluetische Mittel, neben unzähligen anderen, medikamentösen und physikalischen Behandlungsmethoden ohnejeden Erfolg: versucht worden sind.

Schwierig gestaltete sich nun die Frage der Therapie, und sie konnte zunächst nur eine symptomatische sein, da der Prozeß selbst nicht beeinflußt werden kann. Wir entschlossen uns daher zunächst zu einer Keilosteotomie, an der am schärfsten gebogenen Stelle des Knochens; in der Absicht, dadurch die Verkrümmung zu beseitigen und die Tibia etwas zu verkürzen. Die Operation wurde von Herrn Geheimrat $S$ ch e d e ausgeführt, und brachte baldige, wenn auch nicht dauernde Besserung. Die Osteotomie heilte schnell und glatt, und gab dem Bein eine ziemlich gerade Richtung wieder. An dem Röntgenbilde (Fig. 4) erkannte man deutlich die alte Osteotomiestelle. Schon bei dieser ersten Operation fiel der Bau des Knochens selur auf; zu unserem Erstaunen erwies sich der Knochen, den wir als äußerst hart und sklerosiert vermutet hatten, als ein so weiches Gewebe, dal er bequem mit dem Messer geschnitten werden konnte; das überraschte besonders, da es sich um die Diaphyse handelte; die Corticalis, die sonst in diesem Alter fast $1 / 2 \mathrm{~cm}$ stark und splitternd hart zu sein pflegt, war völlig geschwunden, und an das 
Periost grenzte sofort eine spongiöse Substanz, deren Markräume teils von Bindegewebe, teils von Fettmark erfüllt waren. Die einzelnen Lamellen dieser Substanz wurden nach dem Innern des Knochens zu immer spärlicher, und endlich war der Knochen von normal aussehendem Fettmark erfüllt. Unsere Befürchtungen, daß dieser schwer veränderte Knochen schlecht heilen würde, bestätigten sich nicht; die Patientin konnte bald, nach reaktionslosem Verlauf der Osteotomie, mit einer Schienenhülse entlassen werden, und ging wesentlich besser als zuvor. Freilich traten bald wieder Schmerzen auf, jetzt war es in erster Linie das Kniegelenk, welches die Beschwerden verschuldete. Eine zunehmende deformierende Arthritis gestattete nur noch ganz minimale Bewegungen, und diese waren so schmerzhaft und wurden auch durch die Schienenhülse nicht vollständig genug ausgeschaltet, sodal uns eine Resektion des Kniegelenks das beste zu sein schien. Schon damals erwogen wir die Frage einer Amputation, und betrachteten die Resektion nur als einen Versuch. An die Resektion schloß sich aus unbekannten Gründen eine endlose Eiterung an, die Knochenenden zeigten keinerlei Neigung zur Konsolidation, dazu kam, dal auch die Verkrïmmung der Tibia wieder im Zunehmen begriffen war. So willigte die Kranke gern in die Amputation ein, die sie jetzt endgültig von ihren Qualen befreit hat, etwa $3 / 4$ Jahre nach der ersten Operation.

Schon bei der Keilosteotomie war Material zur histologischen Untersuchung gewonnen, ebenso bei der Resektion des

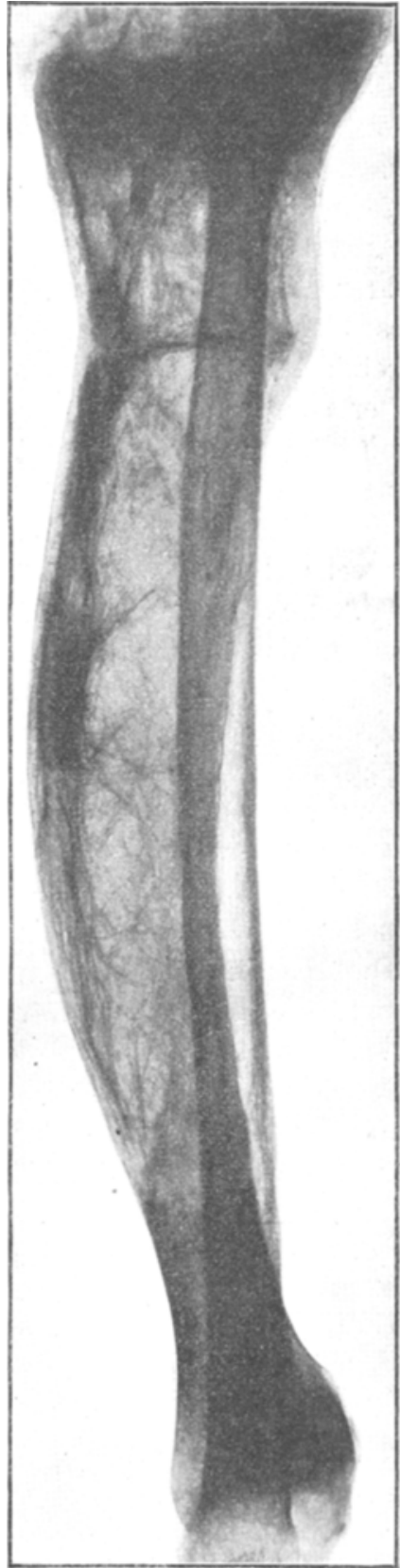

Fig. 4. 
Kniegelenkes. Endlich konnte an dem auspräparierten Knochen nach der Amputation festgestellt werden, daß die Corticalis überall fehlte, man kann den weichen Knochen mit dem Fingernagel eindrücken; spielend leicht gelingt es, den ganzen Knochen mit einer einfachen Säge der Länge nach zu halbieren. Hierbei bemerkt man, daß in der Mitte der Diaphyse das Mark stellenweise einen roten, lymphoiden Charakter trägt. Ganz besonders weich ist die Hinterwand der Tibia, scheinbar gänzlich ihrer Kalksalze beraubt. Das Periost zieht sich ziemlich leicht ab, es bleiben dabei einige dünne Schichten, offenbar periostal neugebildeter, weicher Knochensubstanz an der Knochenhaut hängen. Der noch unaufgesägte, ausgelöste Knochen, ist in der Abbildung (Fig. 4) mit Röntgenstrahlen photographiert dargestellt. Hier tritt deutlich zutage, daß die untere. Epiphyse von der Erkrankung verschont geblieben ist. Deutlich sieht man die alte Osteotomiestelle, an der sich zwecks Heilung derselben relativ viel kalkhaltige Spongiosa gebildet hat. Sehr wichtig und interessant ist nun das mikroskopische Verhalten, das sich im wesentlichen mit den Beobachtungen von Recklinghausen und Stilling deckt. Es handelt sich um eine Osteomyelitis fibrosa, d. b. um eine diffuse Verödung des Fettmarks, mit Umwandlung in Bindegewebe. Das eigentümliche dieser Krankheit besteht jedoch darin, da\} der normale Knochen in gewaltigem Umfang aufgezehrt wird, während sich neuer Knochen nur spärlich und dürftig bildet, meist ohne Ansetzen von Kalksalzen. Daß Neubildung in der Tat stattfindet, das geht aus der Vergrößerung des ganzen Knochens hervor, und zwar findet nicht nur periostale Neubildung statt, die den Knochen verdickt, sondern es findet auch zentrales Wachstum, an den Epiphysenlinien sowohl, wie auch diffus im ganzen Knochen statt. Darauf deutet die gewaltige Verkrümmung aller kranken Teile des Knochens hin. Mikroskopisch jedoch überwiegen stark die Elemente des Abbaues gegen die des Aufbaues. Unzählige Mengen von Riesenzellen, in How sh ip schen Lakunen gelegen (vergl. Fig. 5 Taf. III), beschäftigen sich mit der Resorption des Knochens, indessen das Mark zugrunde geht. Entsprechend dem exquisit-chronischen Verlauf des Leidens sieht man nur noch an ganz wenigen Stellen frischere Entzündungsherde; der unter Fig. 5 Taf. III abgebildete mikroskopische Schnitt ist von der oberen Epiphyse des Knochens gewählt, so daß wir die sekundär zerstörte Gelenkfläche mit darauf erkennen können.

Von der Ätiologie des Leidens ist auch in unserem Falle nichts auszusagen. Mir will es erscheinen, als ließe sich die Pagetsche Erkrankung nicht so scharf von den anderen oben genannten, ähn- 
lichen Prozessen im Knochen absondern. Es handelt sich so ziemlich um das Gleiche, nur ist die Ursache noch unklar; sie ist aber jedenfalls auch nicht wesentlich verschieden von anderen chronischen Infektionskrankheiten der Knochen. Eine bakteriologische Untersuchung wurde mit negativem Ergebnis ausgeführt.

Bezüglich der Therapie kann nur bestätigt werden, was Schu chardt sagt: „Vor chirurgischen Eingriffen zum Ausgleich der Deformitäten kann bei der schweren Ernährungsstörung des Knochensystems im allgemeinen nur dringend gewarnt werden." Freilich heilte unsere erste Osteotomie ja vortrefflich; der Erfolg war vorübergehend ein guter, aber die spätere Resektion des Kniees führte zu hartnäckiger Eiterung, die garnicht zu beseitigen war, und deren Ursache in dem Grundleiden selbst liegen dürfte. Eine Amputation erscheint nach unserem Fall durchaus ratsam, sobald man nachweisen kann, daß nur der eine Knochen krank ist, und sobald die Beschwerden, wie hier, einen Grad erreichen, daß die Patienten sich selbst nach Abnahme des lästigen und unbrauchbaren Gliedes sehnen.

Die Literatur zur Pagetschen Knochenkrankheit findet sich vollständig bei Schuchardt, Krankheit der Knochen und Gelenke, S. 50 .

\section{Erklärung der Abbildungen im Text und auf Tafel III.}

Fig. 1 u. 2. Vordere und seitliche Ansicht der Beine der Patientin bei der Aufnahme in die Klinik.

Fig 3. Röntgenbild aus der Mitte des Unterschenkels. (Verkrümmung, Architektur der Spongiosa.)

Fig. 4. Röntgenbild des nach der Amputation ausgelösten Knochens. Fibula gesund, Tibia verkrümmt, Sehwund der Corticalis, alte Osteotomiestelle, untere Epiphyse gesund.

Fig. 5. Mikroskopischer Schnitt durch die Spongiosa der oberen Tibiaepi physe. (Hämatoxylin, Eosin.) Die knorpelige Gelenkfläche ist durch Arthritis deformans größtenteils zerstört, nur bei $K$. ist sie erhalten. Bei $G$. überwuchert Granulationsgewebe die in der Tiefe unregelmäfig versprengten Knorpelreste $K_{\mathbf{1}}$. Die Knochenbälkchen der Spongiosa (S.) sind dicht besetzt mit Riesenzellen $R$. (Osteoklasten), die meist in Howshipschen Lakunen ljegen. Die Knochenbälkchen sind häufig von einer sehr kernreichen Zellschicht $Z$. umgeben. Die Markräume enthalten nur noch an vereinzelten Stellen das normale Fettmark $F$. Meist ist der Markraum verödet und durch Bindegewebe $B$. ersetzt. $B l$. sind BlutgefäBe. 